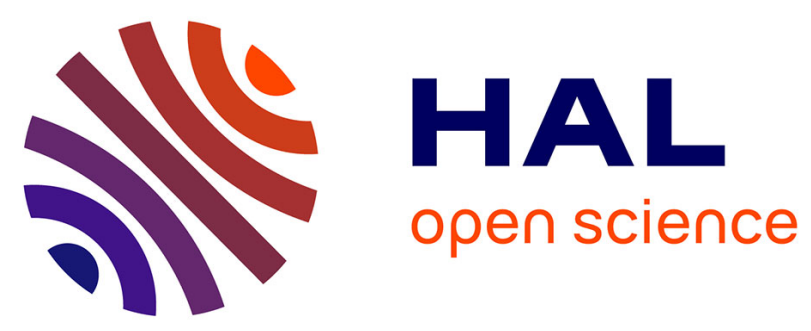

\title{
Asymmetric synthesis of a highly functionalized enantioenriched system close to thapsigargin framework
}

Aurélien Tap, Morgan Jouanneau, Gilles Galvani, Geoffroy Sorin, Marie-Isabelle Lannou, Jean-Pierre Férézou, Janick Ardisson

\section{- To cite this version:}

Aurélien Tap, Morgan Jouanneau, Gilles Galvani, Geoffroy Sorin, Marie-Isabelle Lannou, et al.. Asymmetric synthesis of a highly functionalized enantioenriched system close to thapsigargin framework. Organic \& Biomolecular Chemistry, 2012, 10 (40), pp.8140. 10.1039/c2ob26194d . hal-02370593

\section{HAL Id: hal-02370593 \\ https://hal.science/hal-02370593}

Submitted on 8 Oct 2021

HAL is a multi-disciplinary open access archive for the deposit and dissemination of scientific research documents, whether they are published or not. The documents may come from teaching and research institutions in France or abroad, or from public or private research centers.
L'archive ouverte pluridisciplinaire HAL, est destinée au dépôt et à la diffusion de documents scientifiques de niveau recherche, publiés ou non, émanant des établissements d'enseignement et de recherche français ou étrangers, des laboratoires publics ou privés. 


\title{
Asymmetric synthesis of a highly functionalized enantioenriched system close to thapsigargin framework $\uparrow$
}

\author{
Aurélien Tap, ${ }^{a}$ Morgan Jouanneau, ${ }^{b}$ Gilles Galvani, ${ }^{a}$ Geoffroy Sorin, ${ }^{a}$ Marie-Isabelle Lannou, ${ }^{a}$ \\ Jean-Pierre Férézou ${ }^{b}$ and Janick Ardisson*a
}

A straightforward approach to a highly functionalized enantioenriched bicyclo[5.3.0]decadienone system close to the thapsigargin framework has been achieved. The developed synthetic route involves two main stages: installation of the chains on either side of the quaternary center at $\mathrm{C} 7$ starting from a central enantiopure epoxide and formation of the bicyclic octahydroazulene through subsequent Pauson-Khand annelation.

\section{Introduction}

In recent years, research on natural sesquiterpene lactones has fostered a renewed interest. These lactones are promising candidates in cancer drug discovery and their activities position them as lead compounds in clinical trials. ${ }^{1}$

Thapsigargin ( $\mathrm{Tg}$ ) $\mathbf{1}$ is a highly oxygenated sesquiterpene lactone belonging to the 6,12-guaianolide family, isolated from the Mediterranean plant species Thapsia (Apiaceae). ${ }^{2}$ This compound presents a challenging complex chemical structure, including a polyoxygenated 5-7-5 tricyclic core possessing eight stereogenic centres and functionalized with five different ester groups (Fig. 1). Tg is a very potent inhibitor of the endo/sarcoplasmic calcium ATPase (SERCA). ${ }^{3,4} \mathrm{Tg}$ derivatives are currently under clinical trials for treatment of prostate cancer.

Two main challenges emerge for Tg total synthesis: (i) the construction of the 5,7-fused bicyclic core, (ii) the installation of

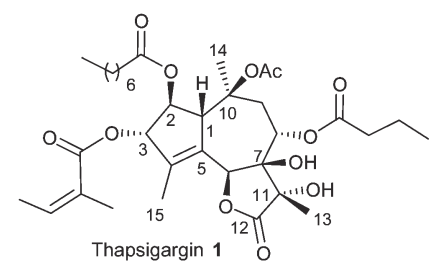

Fig. 1 Thapsigargin (Tg) 1.

\footnotetext{
${ }^{a}$ Faculté de Pharmacie, CNRS UMR 8638, Université Paris Descartes, 4 avenue de l'Observatoire, 75270 Paris Cedex 06, France. E-mail: janick.ardisson@parisdescartes.fr; Tel: +33 (0) 153739754 ${ }^{b}$ LCPSN, ICMMO, CNRS UMR 8182, Bâtiment 410, Université Paris-Sud-11, 15 rue Georges Clemenceau, F-91405 Orsay Cedex, France. Tel: +33 (0)1 69157630
}

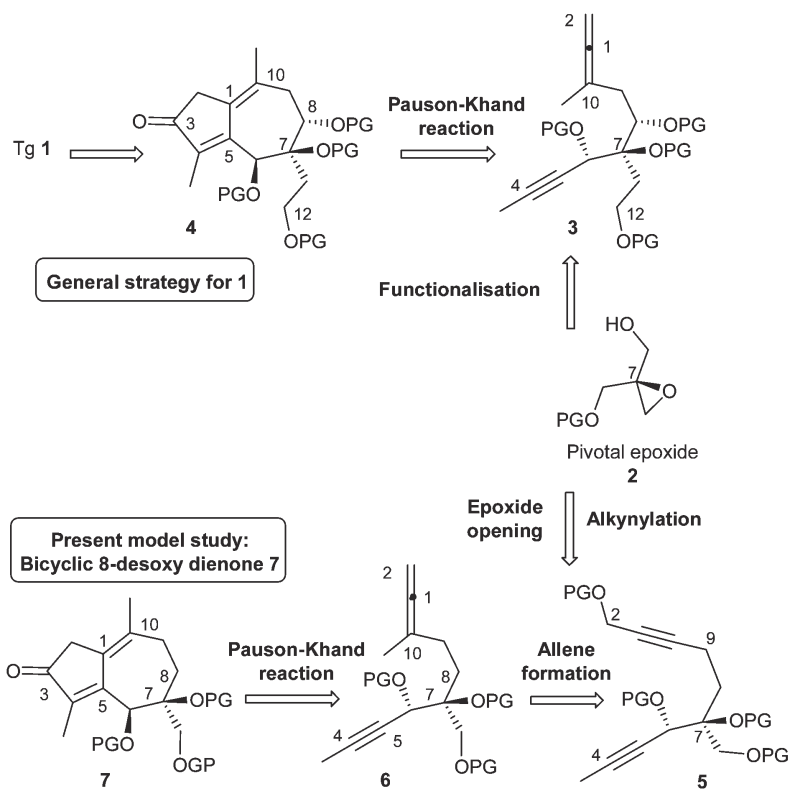

Scheme 1 Synthetic strategy to $\operatorname{Tg} 1$ and 8-desoxy dienone 7.

the dense array of functional groups present throughout the cyclic system. Remarkably, the Ley group succeeded in the first and unique total synthesis of $\mathrm{Tg}^{5}$ as well as in the elaboration of a pivotal scaffold allowing access to unnatural analogues, ${ }^{6}$ by means of a key diene ring closure metathesis step. A few other efforts have led to alternative interesting approaches, through either an efficient domino metathetic route (Kaliappan) ${ }^{7}$ or a photochemical rearrangement (Massanet). ${ }^{8}$

Our general strategy for the synthesis of $\mathrm{Tg} \mathbf{1}$ relies on two key points (Scheme 1). The stereocontrolled installation of the functionalities on either side of the $\mathrm{C} 7$ tertiary alcohol is envisioned at the beginning of the synthesis, through the addition of 
side arms to the pivotal 7-centered $(R)$-epoxide $2,{ }^{9}$ to deliver intermediate 3. Then, Rh(I)-catalyzed allenic Pauson-Khand (A.P.K.) reaction, should allow the construction of bicyclo[5.3.0]decadienone 4 , chosen as an advanced precursor of $\operatorname{Tg} 1 .^{10,11}$

To validate the above strategy, we decided to synthesize the enantioenriched 8-desoxy dienone 7, structurally close to dienone 4. As for $\operatorname{Tg} \mathbf{1}$, the synthetic route will start from $(R)$ epoxide 2, however, with a reduced number of steps. Bicyclic dienone 7 should be accessible from regiocontrolled epoxide opening through propargylation, ${ }^{12}$ followed by successive alkynylation ${ }^{13}$ and A.P.K. reaction, ${ }^{10,11}$ via intermediates 5 and $\mathbf{6}$.

We report here the results of this chemical validation study. ${ }^{14}$

\section{Results and discussion}

The synthesis began with the formation of the known $(R)$-epoxide 10 available by means of an asymmetric Sharpless epoxidation reaction of monoprotected allylic diol 9 with L-(+)-diethyl tartrate [L-(+)-DET], in 75\% yield and up to $90 \%$ ee (Scheme 2). ${ }^{9 a}$ Trityl (Tr) ether 9 was readily prepared in three steps from commercial phosphonate $\mathbf{8}^{15,16}$

The ensuing nucleophilic opening of the epoxide moiety with propargylmagnesium bromide proceeded in high yield to deliver the terminal alkyne 11. ${ }^{12,17,18}$ The latter was then converted into the corresponding PMB acetal before subsequent formylation of the triple bond and protection of the incipient primary hydroxyl group to give the fully protected intermediate $\mathbf{1 2}$ bearing orthogonally protected hydroxyl functions.

The synthesis was continued by installing the side chain at C4-C5 (Scheme 3). Reduction of acetal 12 with DIBAL-H in $\mathrm{CH}_{2} \mathrm{Cl}_{2}$ at low temperature provided the required primary alcohol 13 as the main product in $61 \%$ yield along with the corresponding regioisomeric tertiary alcohol $\mathbf{1 4}$ which was separated by flash chromatography $(12 \%$ yield $) .{ }^{19}$ Then, the expected $(6 S)$ alcohol 15 was obtained through a four-step sequence. Oxidation of alcohol 13 to the corresponding aldehyde using DessMartin conditions ${ }^{20}$ followed by nucleophilic addition of propynyl magnesium bromide afforded a $1: 1$ mixture of the two epimeric propargylic alcohols $\mathbf{1 5}$. Successive oxidation reaction ${ }^{20}$ and reduction of the resulting ynone with $(R)$-Corey-BakshiShibata $[(R)-\mathrm{CBS}]$ reagent delivered the desired $(6 S)$ alcohol $\mathbf{1 5}$ in $78 \%$ yield and a d.r. of $90: 10 .^{13 b, c, 21}$ The absolute
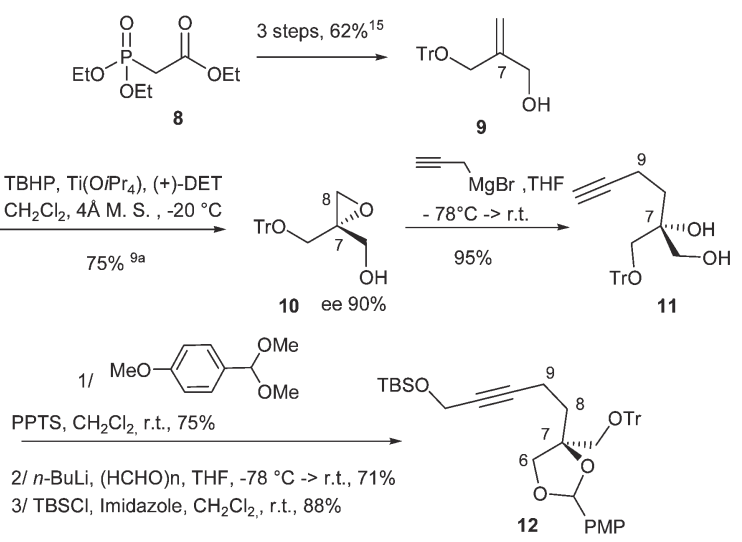

Scheme 2 Synthesis of silyl propargyl ether 12.

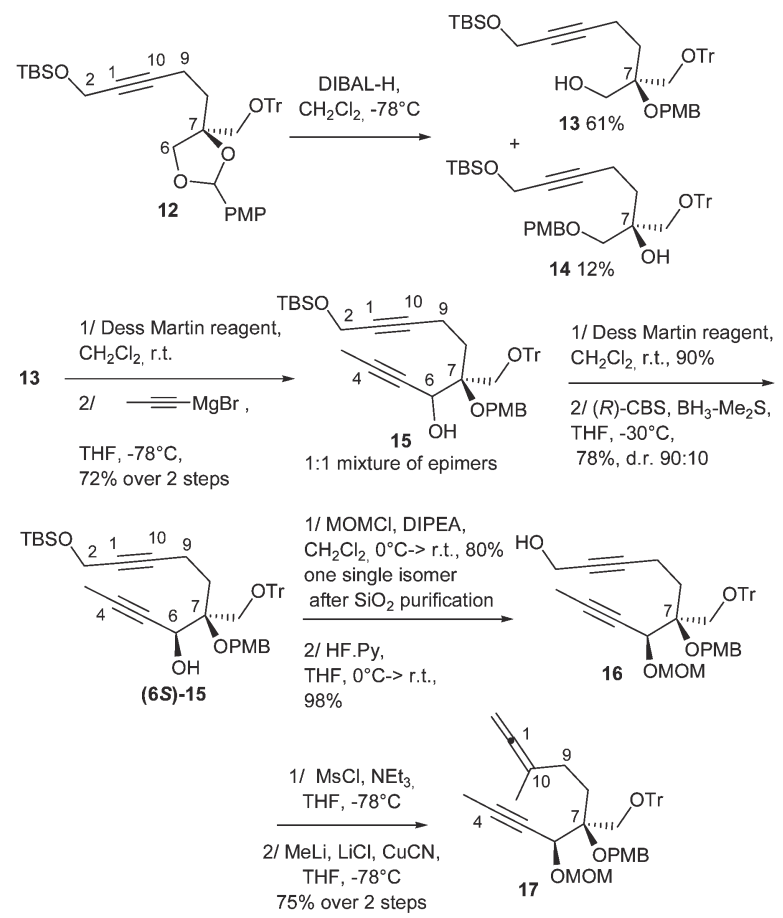

Scheme 3 Synthesis of allenyne 17.

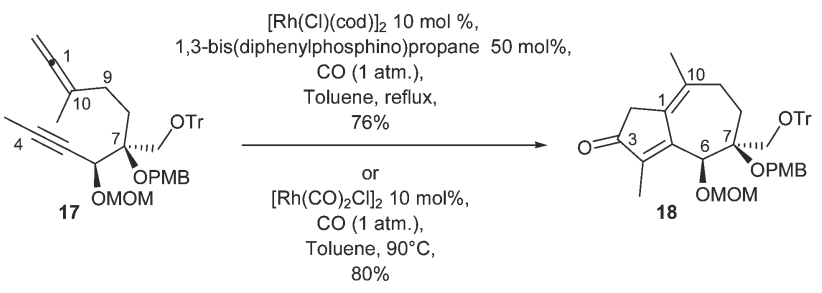

Scheme 4 Synthesis of bicyclo[5.3.0]decadienone $\mathbf{1 8}$.

configuration of the $(S)$-C6 centre of secondary alcohol 15 was confirmed through the synthesis of the corresponding $(R)$ - and (S)- $\alpha$-methoxyphenylacetic acid (MPA) esters. ${ }^{18}$

Subsequent protection of the C6 hydroxyl function of (6S)-15 with a methoxymethyl (MOM) group and selective cleavage of the TBS ether at $\mathrm{C} 2$ allowed isolation of propargyl alcohol $\mathbf{1 6}$ as a single isomer after flash chromatography purification.

Finally, conversion of alcohol $\mathbf{1 6}$ into the allenyl precursor $\mathbf{1 7}$ of P.K.R. annelation was achieved in a two-step sequence: treatment with methanesulfonyl chloride and subsequent $\mathrm{S}_{\mathrm{N}} 2^{\prime}$ reaction with methyl cyanocuprate. ${ }^{10}$

With allenyne $\mathbf{1 7}$ in hand, the feasibility of allene PausonKhand cyclocarbonylation ${ }^{10,11}$ was then investigated under various conditions. ${ }^{21}$ Allenyl compound $\mathbf{1 7}$ was first treated with in situ prepared $\left[\mathrm{Rh}(\mathrm{CO})(\mathrm{dppp})_{2} \mathrm{Cl}\right]-10 \mathrm{~mol} \%$ of $[\mathrm{Rh}(\mathrm{Cl})$ (cod) $]_{2}$ and $50 \mathrm{~mol} \%$ of 1,3-bis(diphenylphosphino)propane under $\mathrm{CO}$ atmosphere in refluxing toluene for 5 hours, to produce the bicyclo[5.3.0] derivative $\mathbf{1 8}$ in $76 \%$ yield (Scheme 4). ${ }^{10}$ Conditions involving the use of $10 \mathrm{~mol} \%$ of commercially available $\left[\mathrm{Rh}(\mathrm{CO})_{2} \mathrm{Cl}\right]_{2}$ in toluene at $90{ }^{\circ} \mathrm{C}$ for 5 hours under a $\mathrm{CO}$ atmosphere were also tested and led to $\mathbf{1 8}$ in $80 \%$ yield; ${ }^{11}$ these latter conditions were preferred for simplicity's sake. 


\section{Conclusion}

In summary, we report here preliminary studies towards a new approach of the synthesis of the highly functionalized bicyclo[5.3.0]decadienone ring system of thapsigargin, starting from the C7-centered enantiopure epoxide $\mathbf{1 0}$ easily accessible by means of an asymmetric Sharpless epoxidation. Around the pivotal starting block $\mathbf{1 0}$ is then articulated the stereocontrolled implementation of the different substituents leading to the intermediate allenyne 17, precursor of the P.K.R. double annelation reaction. The synthetic sequence used to prepare the desired 5-7 bicyclic dienone $\mathbf{1 8}$ allows high and reproducible yields and selectivities.

Application of the present approach to the total synthesis of thapsigargin $\mathbf{1}$ is currently in progress in our laboratories.

\section{Experimental section}

\section{General remarks}

All commercially available reagents and solvents (Fluka, Aldrich, Acros, Fisher) were used without further purification. For reactions requiring anhydrous conditions, commercial dry solvents were directly used (Fluka, Aldrich) or freshly distilled prior to use (THF and $\mathrm{Et}_{2} \mathrm{O}$ over sodium/benzophenone system, DCM and DMSO over calcium hydride and $\mathrm{MeOH}$ and $\mathrm{EtOH}$ over magnesium). Unless otherwise noted, experiments were carried out under argon. Reactions were monitored by TLC (Merck silica gel $60 \mathrm{~F}_{254}$ plates) with detection by use of UV light (254 $\mathrm{nm}$ and $366 \mathrm{~nm}$ ) or a phosphomolybdic acid solution in EtOH (5\%) followed by heating at $100-110{ }^{\circ} \mathrm{C}$. Purifications were performed by flash chromatography on silica gel (Merck

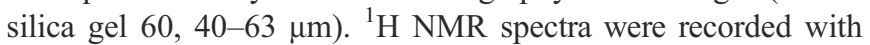
Bruker AVANCE 300 and AVANCE 400 spectrometers at 300 and $400 \mathrm{MHz}$, respectively. Chemical shifts are given in ppm relative to the residual ${ }^{1} \mathrm{H}$ solvent signal $\left(\mathrm{CDCl}_{3}: \delta=7.26 \mathrm{ppm}\right)$ as the internal reference. ${ }^{1} \mathrm{H}$ NMR assignments were confirmed by 2D COSY spectra. The given multiplicities reflect apparent signal patterns. ${ }^{13} \mathrm{C}$ NMR spectra were recorded with the same instruments as above at $100 \mathrm{MHz}$. Chemical shifts are given in ppm relative to the residual ${ }^{13} \mathrm{C}$ solvent signal $\left(\mathrm{CDCl}_{3}: \delta=\right.$ $77.0 \mathrm{ppm}) .{ }^{13} \mathrm{C}$ NMR assignments were confirmed by 2D HSQC spectra. Coupling constants $(J)$ are given in $\mathrm{Hz}$ for all NMR spectroscopic data. Melting points were measured with a Büchi 510 apparatus and are uncorrected. IR spectra were recorded with a Perkin Elmer Spectrum 65 FT-IR spectrometer. Mass spectra were recorded with the following instruments: ESI-MS: Waters ZQ 2000 spectrometer and ESI-TOF-HRMS: Waters LCT Premier spectrometer.

2-Triphenylmethoxymethyl-2-propenol 9 was synthesized according to a reported procedure, starting from ethyl 2-(triphenylmethoxymethyl)propenoate. ${ }^{15}$

(2R)-[2-(Triphenylmethoxymethyl)oxiran-2-yl]methan-1-ol (10). Sharpless epoxidation of $\mathbf{1 3}$ was carried out according to Kang procedure from 2-triphenylmethoxymethyl-2-propenol 9. ${ }^{9 a}[\alpha]_{\mathrm{D}}^{22}$ -20.0 (c 1.5 in $\mathrm{CHCl}_{3}$ ) (lit., -19.5 (c 1.5 in $\mathrm{CHCl}_{3}$ ). Determination of ee via Mosher ester synthesis from $(R)-(+)-\alpha-$-methoxy$\alpha$-(trifluoromethyl)phenylacetic acid [(+)-MTPA] or $(S)-(-)-\alpha-$ methoxy- $\alpha$-(trifluoromethyl)phenyl acetic acid [(-)-MTPA], followed by ${ }^{1} \mathrm{H}$ NMR analysis $\left(400 \mathrm{MHz} ; \mathrm{CDCl}_{3}\right.$ ): ratio of integrals of $\mathrm{CH}_{\mathrm{a}}$ doublets at $\delta 2.73$ and 2.79 corresponds to up to $95: 5$.

(2S)-2-(Trityloxymethyl)hex-5-yne-1,2-diol (11). A mixture of magnesium turnings (4.74 g, $195 \mathrm{mmol})$, mercury(II) chloride $(269 \mathrm{mg}, 0.99 \mathrm{mmol})$ and a single crystal of iodide in freshly distilled diethyl ether $(100 \mathrm{~mL})$ was carefully treated with propargyl bromide ( $80 \%$ in toluene, $10.5 \mathrm{~mL}, 97.4 \mathrm{mmol})$ dissolved in freshly distilled diethyl ether $(40 \mathrm{~mL})$. After the reaction had started, the mixture was cooled to $0{ }^{\circ} \mathrm{C}$ and the rest of the propargyl bromide solution was added within 1 hour. After being cooled at $0{ }^{\circ} \mathrm{C}$ for an additional hour the reaction mixture was warmed to room temperature and stirred for another hour. Epoxide 10 (3.75 g, $10.8 \mathrm{mmol})$ was dissolved in anhydrous THF and cooled to $-78{ }^{\circ} \mathrm{C}$. Under vigorous stirring, this solution was treated with freshly prepared propargylmagnesium bromide $(84 \mathrm{~mL}, 10.84 \mathrm{mmol})$ very slowly within 1 hour and the mixture was slowly warmed to room temperature. After 3 hours the reaction was quenched with saturated aqueous $\mathrm{NH}_{4} \mathrm{Cl}$. The solution was extracted with diethyl ether $(3 \times)$. The combined extracts were dried $\left(\mathrm{MgSO}_{4}\right)$ and concentrated in vacuo. The crude residue was purified by flash chromatography on silica gel (pentane/ethyl acetate $80: 20$ to $75: 25$ ) to yield the expected diol $11(4 \mathrm{~g}, 95 \%)$ as a colorless oil. $[\alpha]_{\mathrm{D}}^{22}-9.6(c 1.5$ in $\left.\mathrm{CHCl}_{3}\right) ; v_{\max } / \mathrm{cm}^{-1} 3418,3296,3061,2929,1491,1449$, $1224,1074,766,708 ; \delta_{\mathrm{H}}\left(400 \mathrm{MHz} ; \mathrm{CDCl}_{3}\right): 7.28-7.44(15 \mathrm{H}$, $\mathrm{m}), 3.61(1 \mathrm{H}, \mathrm{d}, J=11.5 \mathrm{~Hz}), 3.50(1 \mathrm{H}, \mathrm{d}, J=11.5 \mathrm{~Hz}), 3.20$ $(1 \mathrm{H}, \mathrm{d}, J=9.3 \mathrm{~Hz}), 3.12(1 \mathrm{H}, \mathrm{d}, J=9.3 \mathrm{~Hz}), 2.66(1 \mathrm{H}, \mathrm{s})$, $2.22-2.15(2 \mathrm{H}, \mathrm{m}), 1.92(1 \mathrm{H}, \mathrm{t}, J=2.6 \mathrm{~Hz}), 1.81-1.76(2 \mathrm{H}$, m), $1.56(1 \mathrm{H}, \mathrm{s}) ; \delta_{\mathrm{C}}\left(100 \mathrm{MHz} ; \mathrm{CDCl}_{3}\right): 143.4,128.6,128.0$, $127.3,87.0,84.6,73.6,68.5,66.5,66.2,33.3,12.4 ; \mathrm{m} / \mathrm{z}$ $409.1770\left(\mathrm{MNa}^{+}, \mathrm{C}_{26} \mathrm{H}_{26} \mathrm{NaO}_{3}\right.$ requires 409.1779).

(4S)-tert-Butyl\{5-[2-(4-methoxyphenyl)-4-(trityloxymethyl)1,3-dioxolan-4-yl)pent-2-ynyloxy\}dimethylsilane (12). Diol 11 (4.31 g, $11.2 \mathrm{mmol})$ was dissolved in dry $\mathrm{CH}_{2} \mathrm{Cl}_{2}(8 \mathrm{~mL})$ before addition, at room temperature, of $p$-anisaldehyde dimethylacetal $(3.8 \mathrm{~mL}, 22.3 \mathrm{mmol})$ and PPTS $(280 \mathrm{mg}, 1.12 \mathrm{mmol})$. The mixture was stirred at room temperature for 2 hours and the solvent removed under reduced pressure. The crude product was dissolved in $\mathrm{CH}_{2} \mathrm{Cl}_{2}$ before addition of a small quantity of PPTS and a drop of water. The mixture was stirred for $30 \mathrm{~min}$ then quenched with an aqueous saturated solution of $\mathrm{NaHCO}_{3}$ and extracted with $\mathrm{CH}_{2} \mathrm{Cl}_{2}(3 \times)$, the organic layers were combined, washed with brine, dried over $\mathrm{MgSO}_{4}$ and the solvent removed under reduced pressure. The crude residue was dissolved in an anhydrous mixture of THF/MeOH $(50: 2.5 \mathrm{~mL})$. Sodium borohydride $(633 \mathrm{mg}, 16.7 \mathrm{mmol})$ was added at $0{ }^{\circ} \mathrm{C}$. The mixture was stirred $30 \mathrm{~min}$ at $0{ }^{\circ} \mathrm{C}$, quenched with an aqueous saturated solution of $\mathrm{NH}_{4} \mathrm{Cl}$ and extracted with $\mathrm{CH}_{2} \mathrm{Cl}_{2}(3 \times)$; the organic layers were combined, washed with brine, dried over $\mathrm{MgSO}_{4}$ and the solvent removed under reduced pressure. The crude residue was purified by flash chromatography on silica gel (pentane/ethyl acetate $90: 10$ ) to afford (4S)-4-(but-3-ynyl)-2-(4methoxyphenyl)-4-(trityloxymethyl)-1,3-dioxolanes, (4.2 g, $75 \%$, unseparated $1.3: 1$ mixture of diastereomers) as a colorless oil. $v_{\max } / \mathrm{cm}^{-1} 3292,2934,2293,1615,1517,1448,1375,1250$, $1072,1033,918,834,748,704 ; \mathrm{m} / z$ 527.2205 $\mathrm{MNa}^{+}$, 
$\mathrm{C}_{34} \mathrm{H}_{32} \mathrm{NaO}_{4}$ requires 527.2198). Major diastereomer: $\delta_{\mathrm{H}}$ $\left(400 \mathrm{MHz} ; \mathrm{CDCl}_{3}\right)$ : 7.55-7.28 (17 H, m), $6.96(2 \mathrm{H}, \mathrm{d}, J=8.6)$, $5.88(1 \mathrm{H}, \mathrm{s}), 4.07$ (1 H, d, $J=8.5), 3.98(1 \mathrm{H}, \mathrm{d}, J=8.5 \mathrm{~Hz})$, $3.84(3 \mathrm{H}, \mathrm{s}), 3.32(1 \mathrm{H}, \mathrm{d}, J=9.6 \mathrm{~Hz}), 3.26(1 \mathrm{H}, \mathrm{d}, J=$ 9.6 Hz), 2.31-2.27 (1 H, m), 2.19-2.06 (3 H, m), 1.95 (2 H, t, $J$ $=2.7 \mathrm{~Hz}) ; \delta_{\mathrm{C}}\left(100 \mathrm{MHz} ; \mathrm{CDCl}_{3}\right): 160.5,143.6,128.7,128.1$, $128.0,127.8,127.1,113.8,103.9,86.8,84.2,81.9,72.0,68.3$, 65.7, 55.2, 34.9, 13.0. Minor diastereomer: $\delta_{\mathrm{H}}(400 \mathrm{MHz}$; $\left.\mathrm{CDCl}_{3}\right): 7.55-7.28(17 \mathrm{H}, \mathrm{m}), 6.99(2 \mathrm{H}, \mathrm{d}, J=8.6 \mathrm{~Hz}), 5.86$ $(1 \mathrm{H}, \mathrm{s}), 4.20(1 \mathrm{H}, \mathrm{d}, J=8.6 \mathrm{~Hz}), 3.88(1 \mathrm{H}, \mathrm{d}, J=8.6 \mathrm{~Hz}), 3.82$ (3 H, s), 3.23 (2 H, s), 2.31-2.27 (1 H, m), 2.19-2.06 (3 H, m), $1.98(2 \mathrm{H}, \mathrm{t}, J=2.7 \mathrm{~Hz}) ; \delta_{\mathrm{C}}\left(100 \mathrm{MHz} ; \mathrm{CDCl}_{3}\right): 160.4,143.5$, $128.7,128.1,128.0,127.8,127.1,113.6,103.8,86.6,84.2,81.9$, 72.8, 68.4, 65.5, 55.2, 33.9, 13.0 .

A solution of $n$-BuLi (1.6 M in hexane, $1.1 \mathrm{~mL}, 1.75 \mathrm{mmol})$ was added slowly to a stirred solution of the precedent $1.3: 1$ mixture of (4S)-4-(but-3-ynyl)-2-(4-methoxyphenyl)-4(trityloxymethyl)-1,3-dioxolanes $(680 \mathrm{mg}, 1.35 \mathrm{mmol})$ in anhydrous THF $(3 \mathrm{~mL})$, at $-78{ }^{\circ} \mathrm{C}$. The reaction mixture was stirred for 1 hour at $-78{ }^{\circ} \mathrm{C}$ before addition of depolymerized paraformaldehyde (obtained by heating the polymer) (400 $\mathrm{mg}$, $13.5 \mathrm{mmol}$ ). The reaction mixture was stirred for $10 \mathrm{~min}$ at $-78^{\circ} \mathrm{C}$ and then $30 \mathrm{~min}$ at room temperature. The mixture was quenched with an aqueous saturated solution of $\mathrm{NH}_{4} \mathrm{Cl}$ and extracted with $\mathrm{Et}_{2} \mathrm{O}(3 \times)$; the organic layers were combined, washed with brine, dried over $\mathrm{MgSO}_{4}$; then the solvent was removed under reduced pressure. The crude oil was purified by flash chromatography on silica gel (pentane/ethyl acetate $80: 20$ to $70: 30)$ to afford the expected primary alcohol, $(4 S)-5-[2-(4-$ methoxyphenyl)-4-(trityloxymethyl)-1,3-dioxolan-4-yl]pent-2yn-1-ol, (513 mg, 71\%, 1.3:1 mixture of diastereomers), as a colorless oil. $v_{\max } / \mathrm{cm}^{-1} 3519,2943,2253,1615,1518,1448$, 1375, 1250, 1072, 1034, 918, 834, 749, 710; m/z 557.2310 $\left(\mathrm{MNa}^{+}, \mathrm{C}_{35} \mathrm{H}_{34} \mathrm{NaO}_{5}\right.$ requires 557.2304). Major diastereomer: $\delta_{\mathrm{H}}\left(400 \mathrm{MHz} ; \mathrm{CDCl}_{3}\right): 7.50-7.23(17 \mathrm{H}, \mathrm{m}), 6.93(2 \mathrm{H}, \mathrm{d}, J=$ $8.7 \mathrm{~Hz}), 5.83(1 \mathrm{H}, \mathrm{s}), 4.20(2 \mathrm{H}, \mathrm{s}), 4.03(1 \mathrm{H}, \mathrm{d}, J=8.5 \mathrm{~Hz})$, $3.93(1 \mathrm{H}, \mathrm{d}, J=8.5 \mathrm{~Hz}), 3.82(\mathrm{~s}, 3 \mathrm{H}), 3.24(1 \mathrm{H}, \mathrm{d}, J=9.6 \mathrm{~Hz})$, $3.20(1 \mathrm{H}, \mathrm{d}, J=9.6 \mathrm{~Hz}), 2.32-2.19(2 \mathrm{H}, \mathrm{m}), 2.12-1.93(3 \mathrm{H}$, $\mathrm{m}) ; \delta_{\mathrm{C}}\left(100 \mathrm{MHz} ; \mathrm{CDCl}_{3}\right): 160.5,143.6,128.7,128.2,128.1$, $127.9,127.1,113.8,103.9,86.8,86.1,82.0,78.4,72.1,65.7$, 55.3, 51.4, 35.0, 13.3. Minor diastereomer: $\delta_{\mathrm{H}}(400 \mathrm{MHz}$; $\left.\mathrm{CDCl}_{3}\right): 7.50-7.23(17 \mathrm{H}, \mathrm{m}), 6.85(2 \mathrm{H}, \mathrm{d}, J=8.7 \mathrm{~Hz}), 5.80$ $(1 \mathrm{H}, \mathrm{s}), 4.22(2 \mathrm{H}, \mathrm{s}), 4.16(1 \mathrm{H}, \mathrm{d}, J=8.6 \mathrm{~Hz}), 3.82(1 \mathrm{H}, \mathrm{d}$, $J=8.6 \mathrm{~Hz}), 3.80(3 \mathrm{H}, \mathrm{s}), 3.16(2 \mathrm{H}, \mathrm{s}), 2.32-2.19(2 \mathrm{H}, \mathrm{m})$, $2.12-1.93(3 \mathrm{H}, \mathrm{m}) ; \delta_{\mathrm{C}}\left(100 \mathrm{MHz} ; \mathrm{CDCl}_{3}\right): 160.5,143.6,128.7$, $128.2,128.1,127.9,127.1,113.7,103.8,86.8,86.1,82.0,78.4$, 72.9, 65.5, 55.3, 51.4, 34.0, 13.3.

TBDMSCl (558 mg, $3.71 \mathrm{mmol})$ and imidazole (526 mg, $7.73 \mathrm{mmol}$ ) were successively added to a stirred solution of the precedent mixture of alcohols $(1.65 \mathrm{~g}, 3.09 \mathrm{mmol})$ in anhydrous $\mathrm{CH}_{2} \mathrm{Cl}_{2}(20 \mathrm{~mL})$, at room temperature. The mixture was stirred for 1 hour at room temperature, quenched with an aqueous saturated solution of $\mathrm{NH}_{4} \mathrm{Cl}$ and extracted with $\mathrm{CH}_{2} \mathrm{Cl}_{2}(3 \times)$; the organic layers were combined, washed with brine, dried over $\mathrm{MgSO}_{4}$ and the solvent removed under reduced pressure. The crude residue was purified by flash chromatography on silica gel (pentane/ethyl acetate $98: 2$ to $95: 5$ ) to yield the expected ether 12 (1.9 g, 95\%, 1.3: 1 mixture of diastereomers), as a colorless oil. $v_{\max } / \mathrm{cm}^{-1} 2928,2856,1615,1517,1448,1249,1071,834$,
777, 703; $m / z \quad 671 \quad\left(\mathrm{MNa}^{+}\right) ; \quad m / z \quad 671.3188 \quad\left(\mathrm{MNa}^{+}\right.$, $\mathrm{C}_{41} \mathrm{H}_{48} \mathrm{NaO}_{5} \mathrm{Si}$ requires 671.3169). Major diastereomer: $\delta_{\mathrm{H}}$ $\left(400 \mathrm{MHz} ; \mathrm{CDCl}_{3}\right): 7.49-7.25(17 \mathrm{H}, \mathrm{m}), 6.91(2 \mathrm{H}, \mathrm{d}, J=$ $8.7 \mathrm{~Hz}), 5.81(1 \mathrm{H} \mathrm{s}), 4.27(2 \mathrm{H}, \mathrm{s}), 4.01(1 \mathrm{H}, \mathrm{d}, J=8.5 \mathrm{~Hz})$, $3.90(1 \mathrm{H}, \mathrm{d}, J=8.5 \mathrm{~Hz}), 3.82(3 \mathrm{H}, \mathrm{s}), 3.22(1 \mathrm{H}, \mathrm{d}, J=$ $9.6 \mathrm{~Hz}), 3.15(1 \mathrm{H}, \mathrm{d}, J=9.6 \mathrm{~Hz}), 2.30-2.20(2 \mathrm{H}, \mathrm{m})$, 2.09-2.00 (2 H, m), $0.91(9 \mathrm{H}, \mathrm{s}), 0.11(6 \mathrm{H}, \mathrm{s}) ; \delta_{\mathrm{C}}(100 \mathrm{MHz}$; $\left.\mathrm{CDCl}_{3}\right): 160.5,143.6,128.7,128.2,128.1,127.9,127.1,113.8$, $103.9,86.8,84.9,82.0,78.8,72.1,65.7,55.3,51.9,35.1,34.0$, 25.9 13.4, -5.1. Minor diastereomer: $\delta_{\mathrm{H}}\left(400 \mathrm{MHz} ; \mathrm{CDCl}_{3}\right)$ : 7.49-7.25 (17 H, m), 6.85 (2 H, d, $J=8.6 \mathrm{~Hz}), 5.80$ (1 H, s), 4.29 (2 H, s), $4.13(1 \mathrm{H}, \mathrm{d}, J=8.6 \mathrm{~Hz}), 3.83(1 \mathrm{H}, \mathrm{d}, J=$ $8.6 \mathrm{~Hz}), 3.80(3 \mathrm{H}, \mathrm{s}), 3.16(2 \mathrm{H}, \mathrm{s}), 2.30-2.20(2 \mathrm{H}, \mathrm{m})$, 2.09-2.00 (2 H, m), $0.92(9 \mathrm{H}, \mathrm{s}), 0.12(6 \mathrm{H}, \mathrm{s}) ; \delta_{\mathrm{C}}(100 \mathrm{MHz}$; $\left.\mathrm{CDCl}_{3}\right): 160.5,143.6,128.7,128.2,128.1,127.9,127.1,113.7$, $103.9,86.8,84.9,82.0,78.8,72.8,65.6,55.3,51.9,35.1,34.0$, $25.913 .4,-5.1$.

(2R)-7-(tert-Butyldimethylsilyloxy)-2-(4-methoxybenzyloxy)-2(trityloxymethyl)hept-5-yn-1-ol (13) and (2R)-7-(tert-butyldimethylsilyloxy)-1-(4-methoxybenzyloxy)-2-(trityloxymethyl)hept5-yn-2-ol (14). DIBAL-H (1.1 M in cyclohexane, $4.2 \mathrm{~mL}$, $4.67 \mathrm{mmol}$ ) was added at $-78{ }^{\circ} \mathrm{C}$ over $3.5 \mathrm{~h}$ to a stirred solution of the PMP acetal 12 (605 $\mathrm{mg}, 0.934 \mathrm{mmol})$ in anhydrous $\mathrm{CH}_{2} \mathrm{Cl}_{2}(10 \mathrm{~mL})$. The mixture was stirred for 1 hour at $-78{ }^{\circ} \mathrm{C}$, quenched with an aqueous solution of $\mathrm{NaOH} 4 \mathrm{M}$ and extracted with $\mathrm{CH}_{2} \mathrm{Cl}_{2}(3 \times)$; the organic layers were combined, washed with brine, dried over $\mathrm{MgSO}_{4}$ and the solvent removed under reduced pressure. The crude residue was purified by flash chromatography on silica gel (pentane/ethyl acetate $98: 2$ to $95: 5$ ) to yield in order of elution the required primary alcohol 13, (370 $\mathrm{mg}, 61 \%$ ) and the secondary alcohol 14 (73 $\mathrm{mg}, 12 \%)$ as colorless oils. 13: $[\alpha]_{\mathrm{D}}^{22}-5.9\left(c 1.0\right.$ in $\left.\mathrm{CHCl}_{3}\right) ; v_{\max } / \mathrm{cm}^{-1} 3476$, $2929,2857,1613,1514,1249,1076,836,777,707 ; \delta_{\mathrm{H}}$ (400 MHz; $\left.\mathrm{CDCl}_{3}\right)$ : 7.46-7.17 (17 H, m), $6.82(2 \mathrm{H}, \mathrm{d}, J=$ $8.7 \mathrm{~Hz}), 4.36(1 \mathrm{H}, \mathrm{d}, J=10.5 \mathrm{~Hz}), 4.32(1 \mathrm{H}, \mathrm{d}, J=10.5 \mathrm{~Hz})$, $4.27(2 \mathrm{H}, \mathrm{s}), 3.79(3 \mathrm{H}, \mathrm{s}), 3.74(2 \mathrm{H}, \mathrm{d}, J=6.9 \mathrm{~Hz}), 3.31(1 \mathrm{H}$, d, $J=9.6 \mathrm{~Hz}), 3.07(1 \mathrm{H}, \mathrm{d}, J=9.6 \mathrm{~Hz}), 2.21-2.18(1 \mathrm{H}, \mathrm{m})$, 2.09-1.92 (3 H, m), 1.87 (1 H, t, $J=6.9 \mathrm{~Hz}), 0.91$ (9 H, s), 0.11 $(6 \mathrm{H}, \mathrm{s}) ; \delta_{\mathrm{C}}\left(100 \mathrm{MHz} ; \mathrm{CDCl}_{3}\right): 159.1,143.5,130.6,129.1$, $128.7,127.9,127.1,113.8,86.8,85.2,78.7,78.6,63.9,63.7$, 63.6, 55.3, 51.9, 29.9, 25.9, 12.4, -5.1; $\mathrm{m} / z 673.3318\left(\mathrm{MNa}^{+}\right.$, $\mathrm{C}_{41} \mathrm{H}_{50} \mathrm{NaO}_{5} \mathrm{Si}$ requires 673.3325). 14: $[\alpha]_{\mathrm{D}}^{22}+1.9(c \quad 0.62$ in $\left.\mathrm{CHCl}_{3}\right) ; v_{\max } / \mathrm{cm}^{-1} 3554,2928,1514,1449,1249,835,775 ; \delta_{\mathrm{H}}$ $\left(400 \mathrm{MHz} ; \mathrm{CDCl}_{3}\right): 7.42-7.18(17 \mathrm{H}, \mathrm{m}), 6.86(2 \mathrm{H}, \mathrm{d}, J=8.6$ $\mathrm{Hz}), 4.47(2 \mathrm{H}, \mathrm{s}), 4.25(2 \mathrm{H}, \mathrm{s}), 3.81(3 \mathrm{H}, \mathrm{s}), 3.58(1 \mathrm{H}, \mathrm{d}, J=$ $9.0 \mathrm{~Hz}), 3.47(1 \mathrm{H}, \mathrm{d}, J=9.0 \mathrm{~Hz}), 3.14(1 \mathrm{H}, \mathrm{d}, J=8.8 \mathrm{~Hz}), 3.04$ $(1 \mathrm{H}, \mathrm{d}, J=8.8 \mathrm{~Hz}), 2.47(1 \mathrm{H}, \mathrm{s}), 2.19-2.05(2 \mathrm{H}, \mathrm{m}), 1.84(1 \mathrm{H}$, $\mathrm{d}, J=8.3 \mathrm{~Hz}), 1.81(1 \mathrm{H}, \mathrm{d}, J=8.3 \mathrm{~Hz}), 0.90(9 \mathrm{H}, \mathrm{s}), 0.10(6 \mathrm{H}$, s); $m / z 673.3337\left(\mathrm{MNa}^{+}, \mathrm{C}_{41} \mathrm{H}_{50} \mathrm{NaO}_{5} \mathrm{Si}\right.$ requires 673.3325).

(4S,5S)-10-(tert-Butyldimethylsilyloxy)-5-(4-methoxybenzyloxy)5-(trityloxymethyl)deca-2,8-diyn-4-ol (6S-15). Dess-Martin reagent (509 $\mathrm{mg}, 1.2 \mathrm{mmol}$ ) was added to a stirred solution of $\mathbf{1 3}$ $(520 \mathrm{mg}, 0.8 \mathrm{mmol})$ in anhydrous $\mathrm{CH}_{2} \mathrm{Cl}_{2}(13 \mathrm{~mL})$ at room temperature. The mixture was stirred for $45 \mathrm{~min}$ at room temperature and quenched with an aqueous solution of $\mathrm{Na}_{2} \mathrm{~S}_{2} \mathrm{O}_{3}$ and extracted with $\mathrm{CH}_{2} \mathrm{Cl}_{2}(3 \times)$; the organic layers were combined, washed with brine, dried over $\mathrm{MgSO}_{4}$ and the solvent removed 
under reduced pressure. A solution of propynyl magnesium bromide $(0.5 \mathrm{M}$ in THF, $8.0 \mathrm{mmol}, 16 \mathrm{~mL})$ was added to a stirred solution of the precedent crude aldehyde in anhydrous THF $(1 \mathrm{~mL})$ at $-78^{\circ} \mathrm{C}$. The mixture was warmed to room temperature and stirred for 2 hours. The reaction was quenched with an aqueous solution of $\mathrm{NH}_{4} \mathrm{Cl}$ and extracted with $\mathrm{Et}_{2} \mathrm{O}(3 \times)$; the organic layers were combined, washed with brine, dried over $\mathrm{MgSO}_{4}$ and the solvent removed under reduced pressure. The crude residue was purified by flash chromatography on silica gel (pentane/ethyl acetate $90: 10$ ) to afford the secondary alcohol 15 as a $1: 1$ mixture of epimers (396 $\mathrm{mg}, 72 \%$, yellow oil). DessMartin reagent (492 $\mathrm{mg}, 1.16 \mathrm{mmol}$ ) was then added to a stirred solution of the latter alcohol $(396 \mathrm{mg}, 0.58 \mathrm{mmol})$ in anhydrous $\mathrm{CH}_{2} \mathrm{Cl}_{2}(10 \mathrm{~mL})$ at room temperature. The mixture was stirred for 1 hour at room temperature, then quenched with an aqueous solution of $\mathrm{Na}_{2} \mathrm{~S}_{2} \mathrm{O}_{3}$ and extracted with $\mathrm{CH}_{2} \mathrm{Cl}_{2}(3 \times)$; the organic layers were combined, washed with brine, dried over $\mathrm{MgSO}_{4}$ and the solvent removed under reduced pressure. The crude residue was purified by flash chromatography on silica gel (pentane/ethyl acetate $98: 2$ to $95: 5)$ to afford pure $(5 S)-10$ (tert-butyldimethylsilyloxy)-5-(4-methoxybenzyloxy)-5-(trityloxymethyl)deca-2,8-diyn-4-one, (360 mg, 90\%) as a yellow oil. $[\alpha]_{\mathrm{D}}^{22}-8.7\left(c 1.0\right.$ in $\left.\mathrm{CHCl}_{3}\right) ; v_{\max } / \mathrm{cm}^{-1} 2929,2857,2218,1675$, $1515,1250,1079,837,707 ; \delta_{\mathrm{H}}\left(400 \mathrm{MHz} ; \mathrm{CDCl}_{3}\right): 7.44-7.22$ $(17 \mathrm{H}, \mathrm{m}), 6.83(2 \mathrm{H}, \mathrm{d}, J=8.7 \mathrm{~Hz}), 4.34(1 \mathrm{H}, \mathrm{d}, J=10.2 \mathrm{~Hz})$, 4.27 (2 H, s), 4.20 (1 H, d, $J=10.2 \mathrm{~Hz}), 3.79$ (3 H, s), 3.52 (1 $\mathrm{H}, \mathrm{d}, J=9.5 \mathrm{~Hz}), 3.25(\mathrm{~d}, 1 \mathrm{H}, J=9.5 \mathrm{~Hz}), 2.35-2.13(2 \mathrm{H}, \mathrm{m})$, $2.01(3 \mathrm{H}, \mathrm{s}), 1.86-1.92(2 \mathrm{H}, \mathrm{m}), 0.91(9 \mathrm{H}, \mathrm{s}), 0.11(6 \mathrm{H}, \mathrm{s}) ; \delta_{\mathrm{C}}$ $\left(100 \mathrm{MHz} ; \mathrm{CDCl}_{3}\right): 189.5,159.1,143.1,130.0,129.1,128.8$, $127.8,127.1,113.7,92.8,86.7,85.0,84.5,79.0,78.9,66.0$, $63.2,55.3,51.9,29.5,25.9,12.3,4.38,-5.09 ; \mathrm{m} / \mathrm{z} 709.3333$ $\left(\mathrm{MNa}^{+}, \mathrm{C}_{44} \mathrm{H}_{50} \mathrm{NaO}_{5} \mathrm{Si}\right.$ requires 709.3325).

$\mathrm{BH}_{3}-\mathrm{Me}_{2} \mathrm{~S}(221 \mu \mathrm{L}, 2.33 \mathrm{mmol})$ and $(R)$-Corey-BakshiShibata $[(R)-\mathrm{CBS})]$ reagent $(1 \mathrm{M}$ in toluene, $0.93 \mathrm{~mL}$, $0.93 \mathrm{mmol}$ ) were successively added to a stirred solution of the preceding ketone $(320 \mathrm{mg}, 0.466 \mathrm{mmol})$ in anhydrous THF $(4 \mathrm{~mL})$ at $-30{ }^{\circ} \mathrm{C}$. The mixture was stirred at $-30{ }^{\circ} \mathrm{C}$ for 1 hour and quenched slowly with EtOH and then with water. The aqueous layer was extracted with $\mathrm{Et}_{2} \mathrm{O}(3 \times)$. The organic layers were combined, washed with brine, dried over $\mathrm{MgSO}_{4}$ and the solvent removed under reduced pressure. The crude residue was purified by flash chromatography on silica gel (pentane-ethyl acetate $90: 10)$ to give $(\mathbf{6 S})-\mathbf{1 5}(250 \mathrm{mg}, 78 \%$, d.r. $90: 10)$, as a colorless oil. $v_{\max } / \mathrm{cm}^{-1} 3529,2928,1613,1514,1449,1249$, $1075,836,777,746,707 ; \delta_{\mathrm{H}}\left(400 \mathrm{MHz} ; \mathrm{CDCl}_{3}\right): 7.48-7.16$ $(17 \mathrm{H}, \mathrm{m}), 6.83(2 \mathrm{H}, \mathrm{d}, J=8.6 \mathrm{~Hz}), 4.81(1 \mathrm{H}, \mathrm{dq}, J=5.8$, $2.0 \mathrm{~Hz}), 4.41(1 \mathrm{H}, \mathrm{d}, J=10.5 \mathrm{~Hz}), 4.30(1 \mathrm{H}, \mathrm{d}, J=10.5 \mathrm{~Hz})$, $4.29(2 \mathrm{H}, \mathrm{s}), 3.79(3 \mathrm{H}, \mathrm{s}), 3.41(1 \mathrm{H}, \mathrm{d}, J=9.8 \mathrm{~Hz}), 3.22(1 \mathrm{H}$, $\mathrm{d}, J=9.8 \mathrm{~Hz}), 2.90(1 \mathrm{H}, \mathrm{d}, J=5.8 \mathrm{~Hz}), 2.35-2.06(4 \mathrm{H}, \mathrm{m})$, $1.86(3 \mathrm{H}, \mathrm{d}, J=2 \mathrm{~Hz}), 0.93(9 \mathrm{H}, \mathrm{s}), 0.13(6 \mathrm{H}, \mathrm{s}) ; \delta_{\mathrm{C}}$ (100 MHz; $\left.\mathrm{CDCl}_{3}\right)$ : 159.1, 143.2, 130.5, 129.2, 128.8, 127.9, 127.2, 113.7, 87.2, 85.4, 83.2, 79.5, 78.6, 77.2, 65.9, 64.6, 63.7, 55.3, 52.0, 30.0, 25.9, 12.9, 3.8, -5.1; m/z $711.3490\left(\mathrm{MNa}^{+}\right.$, $\mathrm{C}_{44} \mathrm{H}_{52} \mathrm{NaO}_{5} \mathrm{Si}$ requires 711.3482).

(6S,7S)-6-(4-Methoxybenzyloxy)-7-(methoxymethoxy)-6-(trityloxymethyl)deca-2,8-diyn-1-ol (16). DIPEA (50 $\mu \mathrm{L}, 0.64 \mathrm{mmol})$ and $\mathrm{MOMCl}(130 \mu \mathrm{L}, 0.728 \mathrm{mmol})$ were successively added to a solution of alcohol (6S)-15 (200 $\mathrm{mg}, 0.291 \mathrm{mmol})$ in anhydrous $\mathrm{CH}_{2} \mathrm{Cl}_{2}(2 \mathrm{~mL})$ at $0{ }^{\circ} \mathrm{C}$. The mixture was stirred at room temperature for 4 hours and quenched with water. The aqueous layers were extracted with $\mathrm{CH}_{2} \mathrm{Cl}_{2}(3 \times)$. The organic layers were combined, washed with brine, dried over $\mathrm{MgSO}_{4}$ and the solvent removed under reduced pressure. The crude residue was purified by flash chromatography on silica gel (pentane/ethyl acetate $90: 10$ ) to yield the required pure MOM ether, (5S,6S)-6-(4-methoxybenzyloxy)-13,13,14,14-tetramethyl5-( prop-1-ynyl)-6-(trityloxymethyl)-2,4,12-trioxa-13-silapentadec-9-yne, (170 mg, 80\%, single diastereomer) as a colorless oil. $[\alpha]_{\mathrm{D}}^{22}+33.4\left(c 1.0\right.$ in $\left.\mathrm{CHCl}_{3}\right) ; v_{\max } / \mathrm{cm}^{-1} 2931,1810,1071,905$, 727, 648; $\delta_{\mathrm{H}}\left(400 \mathrm{MHz} ; \mathrm{CDCl}_{3}\right): 7.48-7.18(17 \mathrm{H}, \mathrm{m}), 6.82$ $(2 \mathrm{H}, \mathrm{d}, J=8.6 \mathrm{~Hz}), 4.93(1 \mathrm{H}, \mathrm{d}, J=6.6 \mathrm{~Hz}), 4.73(1 \mathrm{H}, \mathrm{q}, J=$ $2.0 \mathrm{~Hz}), 4.59(1 \mathrm{H}, \mathrm{d}, J=6.6 \mathrm{~Hz}), 4.48(2 \mathrm{H}, \mathrm{s}), 4.28(2 \mathrm{H}, \mathrm{s})$, $3.79(3 \mathrm{H}, \mathrm{s}), 3.41(1 \mathrm{H}, \mathrm{d}, J=9.9 \mathrm{~Hz}), 3.35(3 \mathrm{H}, \mathrm{s}), 3.33(1 \mathrm{H}$, d, $J=9.9 \mathrm{~Hz}), 2.31-2.06(4 \mathrm{H}, \mathrm{m}), 1.82(3 \mathrm{H}, \mathrm{d}, J=2 \mathrm{~Hz}), 0.91$ $(9 \mathrm{H}, \mathrm{s}), 0.12(6 \mathrm{H}, \mathrm{s}) ; \delta_{\mathrm{C}}\left(100 \mathrm{MHz} ; \mathrm{CDCl}_{3}\right): 158.7,143.6$, $131.6,128.9,128.6,127.7,127.0,113.5,94.6,88.0,85.7,83.9$, $79.8,78.3,75.3,69.2,65.0,63.9,56.1,55.3,52.0,31.0,25.9$, $12.9,3.7,-5.1 ; m / z 755.3753\left(\mathrm{MNa}^{+}, \mathrm{C}_{46} \mathrm{H}_{56} \mathrm{NaO}_{6} \mathrm{Si}\right.$ requires 755.3744).

A molar solution of HF.Py $(1.65 \mathrm{~mL}, 1.64 \mathrm{mmol})$ was added to a solution of the preceding silyl ether $(60 \mathrm{mg}, 0.082 \mathrm{mmol})$ in anhydrous THF $(0.6 \mathrm{~mL})$ at $0{ }^{\circ} \mathrm{C}$. The mixture was stirred at $0{ }^{\circ} \mathrm{C}$ for $10 \mathrm{~min}$, warmed to room temperature, then stirred for 1 hour. The reaction was quenched with an aqueous solution of $\mathrm{NaHCO}_{3}$ and extracted with $\mathrm{Et}_{2} \mathrm{O}(3 \times)$. The organic layers were combined, washed with brine, dried over $\mathrm{MgSO}_{4}$ and the solvent removed under reduced pressure. The crude residue was purified by flash chromatography on silica gel (pentane-ethyl acetate $90: 10)$ to afford pure $16(47 \mathrm{mg}, 93 \%)$ as a colorless oil. $[\alpha]_{\mathrm{D}}^{22}$ +34.9 (c 1.0 in $\mathrm{CHCl}_{3}$ ); $v_{\max } / \mathrm{cm}^{-1} 2950,1510,1451,1247$, $1033,755,706 ; \delta_{\mathrm{H}}\left(400 \mathrm{MHz} ; \mathrm{CDCl}_{3}\right): 7.49-7.18(17 \mathrm{H}, \mathrm{m})$, $6.81(2 \mathrm{H}, \mathrm{d}, J=8.6 \mathrm{~Hz}), 4.93(1 \mathrm{H}, \mathrm{d}, J=6.6 \mathrm{~Hz}), 4.79(1 \mathrm{H}$, q, $J=2.1 \mathrm{~Hz}), 4.58(1 \mathrm{H}, \mathrm{d}, J=6.6 \mathrm{~Hz}), 4.49(2 \mathrm{H}, \mathrm{s}), 4.19$ $(2 \mathrm{H}, \mathrm{s}), 3.79(3 \mathrm{H}, \mathrm{s}), 3.38(1 \mathrm{H}, \mathrm{d}, J=8.8 \mathrm{~Hz}), 3.33(3 \mathrm{H}, \mathrm{s})$, $3.33(1 \mathrm{H}, \mathrm{d}, J=8.8 \mathrm{~Hz}), 2.33-2.02(5 \mathrm{H}, \mathrm{m}), 1.82(3 \mathrm{H}, \mathrm{d}, J=$ $2.1 \mathrm{~Hz}) ; \delta_{\mathrm{C}}\left(100 \mathrm{MHz} ; \mathrm{CDCl}_{3}\right): 158.7,143.6,131.5,128.9$, $128.5,127.7,127.0,113.5,94.5,85.7,83.9,79.8,78.3,75.3$, $69.2,65.0,63.9,56.1,55.3,52.0,31.0,25.9,12.9,3.7,-5.1$; $m / z 641.2868\left(\mathrm{MNa}^{+}, \mathrm{C}_{40} \mathrm{H}_{42} \mathrm{NaO}_{6}\right.$ requires 641.2879).

(((S)-2-(4-Methoxybenzyloxy)-2-((S)-1-(methoxymethoxy)but-2ynyl)-5-methylhepta-5,6-dienyloxy)methanetriyl)tribenzene (17). $\mathrm{NEt}_{3}(11.6 \mu \mathrm{L}, 0.084 \mathrm{mmol})$ and $\mathrm{MsCl}(6.5 \mu \mathrm{L}, 0.084 \mathrm{mmol})$ were successively added to a solution of the primary alcohol $\mathbf{1 6}$ (47 mg, $0.076 \mathrm{mmol}$ ) in anhydrous $\mathrm{CH}_{2} \mathrm{Cl}_{2}(0.5 \mathrm{~mL})$, at $0{ }^{\circ} \mathrm{C}$, then the solution was stirred for 1 hour at room temperature. The mixture was quenched with an aqueous solution of $\mathrm{NH}_{4} \mathrm{Cl}$ and extracted with $\mathrm{CH}_{2} \mathrm{Cl}_{2}(3 \times)$. The organic layers were combined, washed with brine, dried over $\mathrm{MgSO}_{4}$ and the solvent removed under reduced pressure. The residue was passed through a short pad of silica gel to afford crude mesylate. To a solution of $\mathrm{CuCN}$ (27 mg, $0.304 \mathrm{mmol})$ and anhydrous $\mathrm{LiCl}(26 \mathrm{mg}, 0.608 \mathrm{mmol})$ in $\mathrm{THF}(0.3 \mathrm{~mL})$ was gradually added $\mathrm{MeLi}\left(1.6 \mathrm{M}\right.$ in $\mathrm{Et}_{2} \mathrm{O}$, $0.2 \mathrm{~mL}, 0.304 \mathrm{mmol})$ at $-78{ }^{\circ} \mathrm{C}$. Then the reaction mixture was warmed to $-20{ }^{\circ} \mathrm{C}$, and the solids dissolved at this temperature. The reaction mixture was cooled to $-78{ }^{\circ} \mathrm{C}$ again, and the crude mesylate was added to the reaction mixture which was further 
stirred for $1 \mathrm{~h} 30$ at this temperature. The reaction mixture was quenched by addition of saturated aqueous $\mathrm{NH}_{4} \mathrm{Cl}$ and extracted with $\mathrm{Et}_{2} \mathrm{O}(5 \times)$. The organic layers were combined, washed with brine, dried over $\mathrm{MgSO}_{4}$ and the solvent removed under reduced pressure. The crude residue was purified by flash chromatography on silica gel (pentane/ethyl acetate $98: 2$ ) to afford pure allene 17 (35 mg, 75\% over 2 steps) as a colorless oil. $[\alpha]_{\mathrm{D}}^{22}$ +50.9 ( c 1.0 in $\mathrm{CHCl}_{3}$ ); $v_{\max } / \mathrm{cm}^{-1} 2931,1514,1248,1096$, 1033, 704; $\delta_{\mathrm{H}}\left(400 \mathrm{MHz} ; \mathrm{CDCl}_{3}\right): 7.49-7.21(17 \mathrm{H}, \mathrm{m}), 6.81$ $(2 \mathrm{H}, \mathrm{d}, J=8.6 \mathrm{~Hz}), 4.93(1 \mathrm{H}, \mathrm{d}, J=6.6 \mathrm{~Hz}), 4.71(1 \mathrm{H}, \mathrm{q}, J=$ $2.1 \mathrm{~Hz}), 4.60(2 \mathrm{H}, \mathrm{m}), 4.58(1 \mathrm{H}, \mathrm{d}, J=6.6 \mathrm{~Hz}), 4.51(2 \mathrm{H}, \mathrm{s})$, $3.79(3 \mathrm{H}, \mathrm{s}), 3.37(1 \mathrm{H}, \mathrm{d}, J=9.9 \mathrm{~Hz}), 3.36(1 \mathrm{H}, \mathrm{d}, J=$ $9.9 \mathrm{~Hz}), 3.32(3 \mathrm{H}, \mathrm{s}), 2.14-1.94(3 \mathrm{H}, \mathrm{m}), 1.82(3 \mathrm{H}, \mathrm{d}, J=$ $2.1 \mathrm{~Hz}), 1.77-1.72(1 \mathrm{H}, \mathrm{m}), 1.64(3 \mathrm{H}, \mathrm{t}, J=3.0 \mathrm{~Hz}) ; \delta_{\mathrm{C}}$ $\left(100 \mathrm{MHz} ; \mathrm{CDCl}_{3}\right): 205.8,158.7,143.9,131.9,129.2,129.0$, $128.8,127.7,127.0,113.6,99.0,94.6,86.7,83.7,80.5,75.6$, $74.7,69.7,65.1,64.0,56.2,55.3,29.5,26.6,19.2,3.9 ; \mathrm{m} / \mathrm{z}$ $639.3110\left(\mathrm{MNa}^{+}, \mathrm{C}_{41} \mathrm{H}_{44} \mathrm{NaO}_{5}\right.$ requires 639.3086).

(4S,5S)-5-(4-Methoxybenzyloxy)-4-(methoxymethoxy)-3,8dimethyl-5-(trityloxymethyl)-4,5,6,7-tetrahydroazulen-2(1H)-one (18). Protocol 1: Allene $17(17 \mathrm{mg}, 0.028 \mathrm{mmol})$ was dissolved in anhydrous toluene $(0.3 \mathrm{~mL})$ and the solution was evacuated and charged with Ar three times and with $\mathrm{CO}$ three times; then $[\mathrm{RhCl}(\mathrm{cod})]_{2}(1.4 \mathrm{mg}, 0.0028 \mathrm{mmol})$ and 1,3-bis(diphenylphosphino)propane $(5.8 \mathrm{mg}, 0.014 \mathrm{mmol})$ were added. The mixture was refluxed under $\mathrm{CO}$ balloon (1 atm) for $5 \mathrm{~h}$. Most of the solvent was evaporated in vacuo and the resulting concentrated mixture was filtered through a pad of celite (washings with ether). The combined filtrates were evaporated in vacuo. The crude residue was purified by flash chromatography on silica gel (pentane/ethyl acetate $80: 20$ ) to yield the title ketone $\mathbf{1 8}$ $(13.7 \mathrm{mg}, 76 \%)$ as a colorless oil.

Protocol 2: Allene 17 (13 mg, $0.021 \mathrm{mmol})$ was dissolved in anhydrous toluene $(0.3 \mathrm{~mL})$ and the solution was evacuated and charged with argon three times, then with $\mathrm{CO}$ three times before addition of $\left[\mathrm{Rh}(\mathrm{CO})_{2} \mathrm{Cl}\right]_{2}(0.8 \mathrm{mg}, 0.002 \mathrm{mmol})$. The mixture was heated at $90{ }^{\circ} \mathrm{C}$ under $\mathrm{CO}$ balloon $(1 \mathrm{~atm})$ for $5 \mathrm{~h}$. Most of the solvent was evaporated in vacuo and the concentrated mixture filtered through a pad of celite (washings with ether). The combined filtrates were evaporated in vacuo and the crude residue purified by flash chromatography on silica gel (pentaneethyl acetate $80: 20)$ to yield $18(10.3 \mathrm{mg}, 80 \%)$ as a colorless oil. $[\alpha]_{\mathrm{D}}^{22}+36.8\left(c 1.0\right.$ in $\left.\mathrm{CHCl}_{3}\right) ; v_{\max } / \mathrm{cm}^{-1} 2924,2852,1694$, $1611,1513,1449,1247,1149,1097,1033,706 ; \delta_{\mathrm{H}}(400 \mathrm{MHz}$; $\left.\mathrm{CDCl}_{3}\right): 7.48-7.20(17 \mathrm{H}, \mathrm{m}), 6.87(2 \mathrm{H}, \mathrm{d}, J=8.7), 5.18(1 \mathrm{H}$, s), $4.79(1 \mathrm{H}, \mathrm{d}, J=6.7 \mathrm{~Hz}), 4.60(1 \mathrm{H}, \mathrm{d}, J=11.2 \mathrm{~Hz}), 4.58$ $(1 \mathrm{H}, \mathrm{d}, J=11.2 \mathrm{~Hz}), 4.55(1 \mathrm{H}, \mathrm{d}, J=6.7 \mathrm{~Hz}), 3.81(3 \mathrm{H}, \mathrm{s})$, $3.30(1 \mathrm{H}, \mathrm{d}, J=10.3 \mathrm{~Hz}), 3.26(3 \mathrm{H}, \mathrm{s}), 3.06(1 \mathrm{H}, \mathrm{d}, J=$ $10.3 \mathrm{~Hz}), 2.86-2.79(1 \mathrm{H}, \mathrm{d}, J=20.8 \mathrm{~Hz}), 2.63-2.56(1 \mathrm{H}, \mathrm{d}$, $J=20.8 \mathrm{~Hz}), 2.38-2.29(1 \mathrm{H}, \mathrm{m}), 2.05-1.93$ (1 $\mathrm{H}, \mathrm{m})$, $1.88-1.78(2 \mathrm{H}, \mathrm{m}), 1.78(3 \mathrm{H}, \mathrm{s}), 1.67(3 \mathrm{H}, \mathrm{s}) ; \delta_{\mathrm{C}}(100 \mathrm{MHz}$; $\left.\mathrm{CDCl}_{3}\right): 205.4,160.4,158.7,143.2,141.7,135.1,131.7,129.1$, $128.7,128.2,127.7,127.0,113.6,95.9,86.7,80.4,75.6,64.9$, 55.3, 40.3, 31.2, 30.3, 23.9, 8.71; m/z $667.3030\left(\mathrm{MNa}^{+}\right.$, $\mathrm{C}_{42} \mathrm{H}_{44} \mathrm{NaO}_{6}$ requires 667.3036).

$(R)-((S)-2-H y d r o x y-2-(t r i t y l o x y m e t h y l) p e n t-4-y n y l)$ 2-methoxy2-phenylacetate. $(R)-(-)-\alpha$-Methoxyphenylacetic acid $(23 \mathrm{mg}$,
$0.140 \mathrm{mmol}$ ), DMAP (4.0 mg, $0.035 \mathrm{mmol})$ and DCC (29 mg, $0.140 \mathrm{mmol}$ ) were successively added to a solution of diol $\mathbf{1 1}$ (45 mg, $0.116 \mathrm{mmol}$ in anhydrous $\mathrm{CH}_{2} \mathrm{Cl}_{2}(1.5 \mathrm{~mL})$, at room temperature. The mixture was stirred for $20 \mathrm{~min}$ and was filtered over a pad of celite. The solvent was removed under reduced pressure. $^{18} \delta_{\mathrm{H}}\left(400 \mathrm{MHz} ; \mathrm{CDCl}_{3}\right): 7.40-7.25(20 \mathrm{H}, \mathrm{m}), 4.72$ $(1 \mathrm{H}, \mathrm{s}), 4.26(1 \mathrm{H}, \mathrm{d}, J=11.2 \mathrm{~Hz}), 4.19(1 \mathrm{H}, \mathrm{d}, J=11.2 \mathrm{~Hz})$, $3.38(3 \mathrm{H}, \mathrm{s}), 3.06(1 \mathrm{H}, \mathrm{d}, J=9.2 \mathrm{~Hz}), 2.99(1 \mathrm{H}, \mathrm{d}, J=$ $9.2 \mathrm{~Hz}), 2.06-1.97$ (2 H, m), $1.86(1 \mathrm{H}, \mathrm{t}, J=2.6 \mathrm{~Hz})$, $1.75-1.65$ (2 H, m).

(S)-((S)-2-Hydroxy-2-(trityloxymethyl)pent-4-ynyl) 2-methoxy2-phenylacetate. $(S)-(+)-\alpha-M e t h o x y p h e n y l a c e t i c$ acid $(29 \mathrm{mg}$, $0.178 \mathrm{mmol}$ ), DMAP (5.0 $\mathrm{mg}, 0.044 \mathrm{mmol})$ and DCC (37 mg, $0.178 \mathrm{mmol}$ ) were successively added to a solution of diol $\mathbf{1 1}$ (57 mg, $0.148 \mathrm{mmol}$ ) in anhydrous $\mathrm{CH}_{2} \mathrm{Cl}_{2}(1.5 \mathrm{~mL})$, at room temperature. The mixture was stirred for $20 \mathrm{~min}$ and was filtered over a pad of celite. The solvent was removed under reduced pressure. $^{18} \delta_{\mathrm{H}}\left(400 \mathrm{MHz} ; \mathrm{CDCl}_{3}\right): 7.41-7.24(20 \mathrm{H}, \mathrm{m}), 4.67$ (1 H, s), 4.20 (2 H, s), $3.38(3 \mathrm{H}, \mathrm{s}), 3.03(3 \mathrm{H}, \mathrm{s}), 1.99-1.91$ (2 H, m), 1.83 (1 H, t, $J=2.6 \mathrm{~Hz}), 1.63-1.47$ (2 H, m).

(R)-((4S,5S)-10-(tert-Butyldimethylsilyloxy)-5-(4-methoxybenzyloxy)-5-(trityloxymethyl)deca-2,8-diyn-4-yl) 2-methoxy-2phenylacetate. $(R)-(-)-\alpha-M e t h o x y p h e n y l a c e t i c$ acid $(5.6 \mathrm{mg}$, $0.034 \mathrm{mmol})$, DMAP (1.0 mg, $0.0083 \mathrm{mmol})$ and DCC (7 mg, $0.034 \mathrm{mmol})$ were successively added to a solution of alcohol (6S)-15 (19 mg, $0.028 \mathrm{mmol})$ in anhydrous $\mathrm{CH}_{2} \mathrm{Cl}_{2}(1 \mathrm{~mL})$, at room temperature. The mixture was stirred for $20 \mathrm{~min}$ and was filtered over a pad of celite. The solvent was removed under reduced pressure. ${ }^{18} \delta_{\mathrm{H}}\left(400 \mathrm{MHz} ; \mathrm{CDCl}_{3}\right): 7.48-7.14(22 \mathrm{H}, \mathrm{m})$, $6.76(2 \mathrm{H}, \mathrm{d}, J=8.7 \mathrm{~Hz}), 6.00(1 \mathrm{H}, \mathrm{q}, J=2.2 \mathrm{~Hz}), 4.65(1 \mathrm{H}$, s), $4.26(2 \mathrm{H}, \mathrm{s}), 4.21(2 \mathrm{H}, \mathrm{s}), 3.78(3 \mathrm{H}, \mathrm{s}), 3.34(3 \mathrm{H}, \mathrm{s}), 3.30$ $(1 \mathrm{H}, \mathrm{d}, J=9.9 \mathrm{~Hz}), 3.18(1 \mathrm{H}, \mathrm{d}, J=9.9 \mathrm{~Hz}), 2.17-1.96(4 \mathrm{H}$, m), $1.70(3 \mathrm{H}, \mathrm{d}, J=2.2 \mathrm{~Hz}), 0.90(9 \mathrm{H}, \mathrm{s}), 0.11(6 \mathrm{H}, \mathrm{s})$.

$(S)-((4 S, 5 S)-10-($ tert-Butyldimethylsilyloxy)-5-(4-methoxybenzyloxy)-5-(trityloxymethyl)deca-2,8-diyn-4-yl) 2-methoxy-2phenylacetate. $(S)-(+)-\alpha-M e t h o x y p h e n y l a c e t i c$ acid $(5.7 \mathrm{mg}$, $0.035 \mathrm{mmol})$, DMAP (1.0 mg, $0.0087 \mathrm{mmol})$ and DCC (7 mg, $0.035 \mathrm{mmol})$ were successively added to a solution of alcohol (6S)-15 (20 mg, $0.029 \mathrm{mmol})$ in anhydrous $\mathrm{CH}_{2} \mathrm{Cl}_{2}(1 \mathrm{~mL})$, at room temperature. The mixture was stirred for $20 \mathrm{~min}$ and was filtered over a pad of celite. The solvent was removed under reduced pressure. ${ }^{18} \delta_{\mathrm{H}}\left(400 \mathrm{MHz} ; \mathrm{CDCl}_{3}\right): 7.48-7.14(22 \mathrm{H}, \mathrm{m})$, $6.76(2 \mathrm{H}, \mathrm{d}, J=8.7 \mathrm{~Hz}), 6.04(1 \mathrm{H}, \mathrm{q}, J=2.2 \mathrm{~Hz}), 4.79(1 \mathrm{H}$, s), 4.25 (2 H, s), 4.09 (2 H, s), $3.78(3 \mathrm{H}, \mathrm{s}), 3.35$ (3 H, s), 3.20 (2 H, s), 2.21-2.02 (4 H, m), 1.78 (3 H, d, $J=2.2 \mathrm{~Hz}), 0.91$ (9 H, s), $0.11(6 \mathrm{H}, \mathrm{s})$.

\section{Acknowledgements}

A. T. and M. J. thank ANR for doctoral fellowships (ANR Thaser 2010). We thank Elise Prost for assistance with NMR experiments.

\section{Notes and references}

1 A. Ghantous, H. Gali-Muhtasib, H. Vuorela, N. A. Saliba and N. Darwiche, Drug Discovery Today, 2010, 15, 668. 
2 (a) U. S. B. Rasmussen, S. B. Christensen and F. Sandberg, Acta Pharm Suec., 1978, 15, 133; (b) G. Falsone, H. Haddad and D. Wendisch, Arch Pharm., 1986, 319, 372; (c) A. Andersen, C. Cornett, A. Lauridsen, C. E. Olsen and S. B. Christensen, Acta Chem. Scand., 1994, 48, 340; (d) S. B. Christensen, A. Andersen and U. W. Smitt, Prog. Chem. Nat. Prod., 1997, 71, 129; (e) H. Liu, K. G. Jensen, L. M. Tran, M. Chen, L. Zhai, C. E. Olsen, H. Sohoel, S. R. Denmeade, J. T. Isaacs and S. B. Christensen, Phytochemistry, 2006, 67, 2651; $(f)$ A. Pagani, F. Pollastro, S. Spera, M. Ballero, O. Sterner and G. Appendino, Nat. Prod. Commun., 2007, 2, 637; (g) M. Lambert, J. L. Wolfender, D. Stærk, S. B. Christensen, K. Hostettmann and J. W. Jaroszewski, Anal. Chem., 2007, 79, 727. X-ray crystallography: (h) C. Toyoshima, M. Nakasako, H. Nomura and H. Ogawa, Nature, 2000, 405, 647; (i) C. Toyoshima and H. Nomura, Nature, 2002, 418, 605.

3 (a) Y. Furuya, P. Lundmo, A. D. Short, D. L. Gill and J. T. Isaacs, Cancer Res., 1994, 54, 6167; (b) Y. Gong, L. J. Blok, J. E. Perry, J. K. Lindzey and D. J. Tindall, Endocrinology, 1995, 136, 2172.

4 (a) S. B. Christensen, A. Andersen, H. Kromann, M. Treiman, B. Tombal, S. Denmeade and J. T. Isaacs, Bioorg. Med. Chem., 1999, 7, 1273; (b) D. J. V. Grien, L. Antony, S. L. Dalrymple, Y. Xu, S. B. Christensen, S. R. Denmeade and J. T. Isaacs, Mol. Cancer Ther, 2009, 8, 1340; (c) J.-K. Huang, C.-T. Chou, H.-T. Chang, S.-S. Shu, C.-C. Kuo, J.-Y. Tsai, W.-C. Liao, J.-L. Wang, K.-L. Lin, Y.-C. Lu, I.-S. Chen, S.-I. Liu, C.-M. Ho and C.-R. Jan, J. Recept. Signal Transduction, 2011, 31, 247; (d) M. Greiner, B. Kreutzer, S. Lang, V. Jung, A. Cavalié, G. Unteregger, R. Zimmermann and B. Wullich, Prostate, 2011, 71, 1074.

5 (a) M. Ball, S. P. Andrews, F. Wierschem, E. Cleator, M. D. Smith and S. V. Ley, Org. Lett., 2007, 9, 663; (b) S. V. Ley, A. Antonello, E. P. Balskus, D. T. Booth, S. B. Christensen, E. Cleator, H. Gold, K. Hogenauer, U. Hunger, R. M. Myers, S. F. Oliver, O. Simic, M. D. Smith, H. Sohoel and A. J. Woolford, Proc. Natl. Acad. Sci. U. S. A., 2004, 101, 12073.

6 (a) S. F. Oliver, K. Högenauer, O. Simic, A. Antonello, M. D. Smith and S. V. Ley, Angew. Chem., Int. Ed., 2003, 42, 5996; (b) H. Sohoel, T. Liljefors, S. V. Ley, S. F. Oliver, A. Antonello, M. D. Smith, C. E. Olsen, J. T. Isaacs and S. B. Christensen, J. Med. Chem., 2005, 48, 7005; (c) S. P. Andrews, M. M. Tait, M. Balland and S. V. Ley, Org. Biomol. Chem., 2007, 5, 1427.

7 K. P. Kaliappan and R. S. Nandurdikar, Org. Biomol. Chem., 2005, 3, 3613.

8 F. L. Manzano, F. M. Guerra, F. J. Moreno-Dorado, Z. D. Jorge and G. M. Massanet, Org. Lett., 2006, 8, 2879.

9 (a) J.-H. Kang, M. A. Siddiqui, D. M. Sigano, K. Krajewski, N. E. Lewin, Y. Pu, P. M. Blumber, J. Lee and V. E. Marquez, Org. Lett., 2004, 6, 2413; (b) K.-i. Takao, Y. Kojima, T. Miyashita, Y. Kentaro, T. Yamada and K.-i. Tadano, Heterocycles, 2009, 77, 167.

10 For Mukai's synthesis of various guaianolide skeletons through A.P.K. reactions see: (a) C. Mukai, I. Nomura, K. Hyamanishi and M. Hanaoka, Org. Lett., 2002, 4, 1755; (b) C. Mukai, I. Nomura and S. Kitagaki, J. Org. Chem., 2003, 68, 1376; (c) C. Mukai, F. Inagaki, T. Yoshida, K. Yoshitani, Y. Hara and S. Kitagaki, J. Org. Chem., 2005, 70, 7159; (d) T. Hirose, N. Miyakoshi and C. Mukai, J. Org. Chem., 2008, 73, 1061; (e) F. Inagaki, S. Narita, T. Hasegawa, S. Kitagaki and C. Mukai, Angew. Chem., Int. Ed., 2009, 48, 2007; $(f)$ T. Kawamura, F. Inagaki, S. Narita, Y. Takahashi, S. Hirata, S. Kitagaki and C. Mukai, Chem.-Eur.
J., 2010, 16, 5173; (g) Y. Hayashi, K. Ogawa, F. Inagaki and C. Mukai, Org. Biomol. Chem., 2012, 10, 4747.

11 For Brummond's synthesis of various guaianolide skeletons through A.P. K. reactions see: (a) K. M. Brummond, H. Chen, K. D. Fischer, A. D. Kerekes, B. Rickards, P. C. Still and S. J. Geib, Org. Lett., 2002, 4, 1931; (b) K. M. Brummond and D. Gao, Org. Lett., 2003, 5, 3491; (c) K. M. Brummond and D. Chen, Org. Lett., 2008, 10, 705; (d) F. Grillet, C. Huang and K. M. Brummond, Org. Lett., 2011, 13, 6304.

12 J. Ramharter and J. Mulzer, Org. Lett., 2009, 11, 1151.

13 For a direct asymmetric alkynylation, see for instance: (a) D. Boyall, D. E. Frantz and E. M. Carreira, Org. Lett., 2002, 4, 2605; for asymmetric reduction of $\alpha, \beta$-ynones, see for instance: (b) E. J. Corey, R. K. Bakshi and S. Shibata, J. Am. Chem. Soc., 1987, 109, 5551; (c) E. J. Corey and C. J. Helal, Angew. Chem., Int. Ed., 1998, 37, 1986; (d) B. M. Trost, J. L. Gunzner, O. Dirat and Y. H. Rhee, J. Am. Chem. Soc., 2002, 124, 10396.

14 For clarity, thapsigargin carbon numeration has been maintained all over the theoretical part of the paper.

15 (a) J. Villieras and M. Rambaud, Synthesis, 1982, 924; (b) M. Honma, T. Sawada, Y. Fujisawa, M. Utsugi, H. Watanabe, A. Umino, T. Matsumura, T. Hagihara, M. Takno and M. Nakada, J. Am. Chem. Soc., 2003, 125, 2860.

16 In an initial approach, trityl ether was not the optimal protecting group in terms of orthogonality. Various ethers corresponding to 9 were prepared; however, among them, PMB ( $p$-methoxybenzyl) and TBS (tert-butyldimethylsilyl) ethers have proved unstable when they are purified by flash chromatography and TBDPS (tert-butyldiphenylsilyl) ether led to significant lower yields than trityl ether ( $45 \%$ versus $75 \%$ ) when subjected to Sharpless epoxidation.

17 The ee $(90 \%)$ and absolute configuration of 11 have been confirmed again through the synthesis of the corresponding $(R)$ - and $(S)$ - $\alpha$-methoxyphenylacetic acid (MPA) esters, proving the absence of Payne rearrangement.

18 Synthesis of $(R)$ - and $(S)$ - $\alpha$-methoxyphenylacetic acid (MPA) esters: (a) B. M. Trost, D. O. O'Krongly and J. L. Belletire, J. Am. Chem. Soc., 1980, 102, 7595; (b) B. M. Trost and D. P. Curran, Tetrahedron Lett., 1981, 22, 4929; (c) B. M. Trost, J. L. Belletire, P. G. Goldleski, P. G. McDougal, J. M. Balkovec, J. J. Baldwin, M. Christy, G. S. Ponticello, S. L. Varga and J. P. Springer, J. Org. Chem., 1986, 51, 2370 .

19 This modest regioselectivity is surprising but consistent with several literature results. Comparable (or even worse) regioselectivities were reported when the primary alcohol function of similar acetal core was protected by a sterically bulky group (for instance TBDPS). (a) K. J. Hale and J. Cai, Tetrahedron Lett., 1996, 37, 4233; (b) J. Xie and D. A. Hale, Tetrahedron Lett., 2009, 50, 4485; (c) J. Xie, Y. Ma and D. A. Horne, Org. Lett., 2009, 11, 5082; (d) J. Xie and D. A. Horne, Tetrahedron Lett., 2009, 50, 4485; (e) J. Xie, Y. Ma and D. A. Horne, Tetrahedron, 2011, 67, 7485; $(f)$ P. D. O'Connor, C. K. Knight, D. Friedrich, X. Peng and L. A. Paquette, J. Org. Chem., 2007, 72, 1747; (g) S. Raghavan and T. Sreekanth, Tetrahedron Lett., 2007, 48, 9090.

20 D. B. Dess and J. C. Martin, J. Org. Chem., 1983, 48, 4155.

21 Recently, at the time when the present study was in progress in our laboratories, Brummond described the synthesis of racemic 6,12-guaianolide systems relatively close to our targets using an A.P.K. strategy ${ }^{11 d}$. 